\title{
Postoperative delirium: a preventable complication in the elderly surgical patient
}

\author{
Gabriella Bettelli1, Bruno Neuner2 \\ 1 IRCCS INRCA, Ancona, Italy \\ 2 Department of Anesthesiology and Intensive Care Medicine, Charité - Universitätsmedizin Berlin, Germany
}

\begin{abstract}
Postoperative delirium (POD) is an acute organic cerebral disturbance of consciousness and attention in combination with additional cognitive symptoms. It usually develops shortly after surgery and lasts for some hours up to some days. It worsens clinical outcomes, prolongs the hospital stay and leads to negative trajectories of cognitive, emotional and functional outcomes up to month if not years after surgery. There are several known predisposing and precipitating factors. Several of them are influenceable. Offering optimal and safe care for an elderly surgery patient requires a team based approach. Strategies for reducing POD incidence include early detection of risk factors, adaptation of surgical and anaesthesiologic techniques, avoiding certain drugs, optimisation of haemostasis, continuously monitoring of the patients' cognitive status as well as early mobilization and careful management of eventual early signs of POD. If POD is prevented, it's negative trajectories may be likewise anticipated.
\end{abstract}

\section{Introduction}

Postoperative delirium (POD) is a serious and dangerous complication than can cause increased morbidity and mortality after surgery.

Although it may affect any age group, it is most common in older patients and after major, long-lasting surgeries.

During the last years, population aging and the increased need for surgery among the elderly have led to increased absolute numbers of patients with POD. This has caused a relevant burden on both the patients and their families as well as on the health care systems.

Corresponding author: Gabriella Bettelli, IRCCS INRCA, Via della Montagnola 81, 60127 Ancona, Italy. E-mail: gabriella.bettelli@vodafone.it

Key words: Postoperative delirium; surgery; cognitive symptoms.

Received for publication: 26 November 2016

Accepted for publication: 5 December 2016

(C) Copyright G. Bettelli and B. Neuner, 2017

Tipografia PI-ME Editrice, Italy

Monaldi Archives for Chest Disease 2017; 87:842

doi: 10.4081/monaldi.2017.842

This article is distributed under the terms of the Creative Commons Attribution Noncommercial License (by-nc 4.0) which permits any noncommercial use, distribution, and reproduction in any medium, provided the original author(s) and source are credited.
POD is associated with increased morbidity, mortality and need for institutionalization. Very often, patients do not recover previous mental capacity, with severe consequences for both patients and society. Impact of POD is often aggravated due to both lack of knowledge and attention among care givers involved in the process, from the indication for surgery till/to aftercare.

Whereas prevention is the most effective way to reduce its incidence, early detection is the cornerstone for reducing its severity. Considering that different medical disciplines act peri-operatively together on the same patient, a team-based approach should be implemented into daily practice. To date screening for POD shows one of the lowest pass rates when measuring adherence to process-based quality indicators [1].

\section{Literature overview}

\section{Definition and clinical presentation}

According to ICD-10, delirium is defined as a not drug-induced, not alcohol-related nonspecific organic cerebral disturbance of consciousness and attention, perception, thinking, memory, psychomotor behaviour, emotion, and the sleep-wake schedule (F05.0). There is neither an official definition nor an agreement about the length of onset time of POD, but the majority of reports refer to a time frame of 6 hours to 3-5 days after operation. Usually POD lasts for some hours or - when untreated - even for several days. Very early onset of POD within the first minutes or hours after weaning from anaesthesia is sometimes referred to as "emergence delirium". In adults and in the elderly, three forms of POD are described, from "hypoactive" (reduced level of attention, apparent sedation, lethargy,) to "hyperactive" (agitation, inappropriate behaviour, restlessness and hallucination, sometimes hyper-motoric behaviour until aggressiveness) and "mixed" forms [2]. About 10-30\% of all PODs are hyperactive, whereas $50 \%$ are mixed forms and $20-40 \%$ purely hypoactive [3]. Age more than 80 years seems to be a predisposing factor for the hypoactive form [3]. Hypoactive forms are more difficult to diagnose since hypoactive patients do less often interfere with medical treatment and care.

\section{Epidemiology}

According to data from the European Union, total numbers of European inhabitants rose from 505.1 million in 2013 to 506.8 million in 2014 . Overall numbers are expected to increase to 512.5 million in 2020 and to 525.5 million by the middle of the century. By $2014,18.5 \%$ of the European population were $65 \%$ and older and $5.1 \%$ were 80 years and older. The proportion of $\geq 65$-year-old European citizens is also expected to increase, and in 2030 that of Europeans $\geq 80$ years is expected to increase to $7.1 \%$.

In addition to the increase of absolute numbers of old and very old persons, medical and technological progress enables surgeries in patients who would not have been operated on some decades ago. Surgery in the elderly should not be regarded as necessarily coinciding with increased complication rates: in patients aged 75 years and more with good functional status, overall survival was as good as in their non- 
functionally impaired contemporaries [4], when undergoing optimal peri-operative care. On the other hand, POD rates in patients with certain surgical procedures are high: numbers given for POD after hip surgery range from $4 \%$ to $53 \%$. A recent meta-analysis on outcomes after POD in cardiac surgery reported incidence rates between $5.8 \%$ and $45.8 \%$ [5]. POD rates between $15 \%$ and $35 \%$ are reported for gastrointestinal surgery [6].

\section{Risk factors}

A widely accepted model of delirium [7] differentiates predisposing factors (related to the patient himself) that make patients more vulnerable toward POD, and precipitating factors (related to perioperative treatment) that may trigger the delirium onset. A patient with few or no predisposing factors needs a major precipitating factor to develop POD, whereas for a patient with many predisposing factors only a trivial precipitating factor will be a sufficient trigger. Predisposing factors for POD are numerous and frequently overlapping.

In the recent NICE Guideline [8], the strongest predisposing factors were cognitive impairment, followed by age $>65-80$ years, illness severity, infection and sensorial impairment. As the analysis was not restricted to postoperative patients, these finding should only partially be applied to the surgical context.

In a recent guideline on POD issued by the American Geriatric Association [9], predisposing factors for POD included advanced age, cognitive impairment, visual or sensory impairment, functional dependence, alcohol abuse and homeostatic alterations. The ESA guideline on the prevention of POD [10] lists predisposing factors such as age, comorbidities including alcohol use disorders, frailty and pre-operative pain as well as precipitating factors such as preoperative fasting and dehydration, Natrium imbalances, anticholinergics, severe blood loss and cavity surgery.

Some predisposing factors are not changeable but factors such as malnutrition or over-medication are clearly influenceable by appropriate peri-operative strategies. Fast-track surgery and dedicated pathways (Acute Care for the Elderly Units and co-management based models such as ortho-geriatric units) may reduce the incidence of POD [11]. Regarding precipitating factors, the reduction of anticholinergic medication, avoidance of benzodiazepines, monitoring depth of anaesthesia intra-operatively and adequate pain management may help in reducing POD incidence [11]. Likewise, after surgery factors facilitating orientation (non-disrupted day/night circle, application of hearing/reading aids) seems to contribute in POD prevention.

\section{Clinical consequences (mortality, long term outcome)}

POD leads to adverse patients' outcomes, increases hospital length of stay and leads to higher hospital charges and higher postoperative resource use [11]. Several studies described negative cognitive trajectories in patients with POD compared with patients without POD. Cognitive recovery is prolonged in POD patients and not all will regain their preoperative cognitive and behavioural capacity and quality of life $[4,12]$. There is also clear evidence that POD increases overall mortality in hip fracture patients as well as in cardiac surgery and in both elective and emergency surgery [13].

\section{Cornerstone for reducing incidence and severity}

There is agreement among researchers that the best way for reducing POD incidence is preoperative identification of the risk factors and their proactive management. In adjunction, optimal intra- and postoperative management have been recognized to be effective in reducing its duration and severity.

\section{Preoperative measures}

In accordance with the ESA GL [11], preoperative evaluation should carefully search for predisposing factors in order to identify patients at higher risk. Preoperative anaesthesia consultation is probably the best moment for risk factors identification, however this task should be considered more as a "team goal" than a responsibility of a single operator.

Once a patient is recognized to be at higher risk, this information should be shared among the team members (anaesthetists, surgeons and nurses) in order to optimally plan the surgical treatment. Patients at higher risk should be preferably treated by less invasive and less lasting procedures, in accordance with the clinical situation. In comparison with inpatient, outpatient setting, ERAS techniques and fasttrack surgery seem to be accompanied by reduced POD rate.

The family and the patient himself should be adequately informed, in order to plan the best perioperative support the entourage is able to offer, such as continuous presence or frequent visiting.

Optimal preoperative management includes stabilization of associated conditions, elimination of inappropriate/predisposing drugs, control of dehydration and metabolic imbalance and reduced preoperative fasting.

\section{Intraoperative measures}

Whereas there is no evidence that general anaesthesia increases POD risk in comparison with regional techniques, evidence exists that too deep anaesthesia contributes to POD development [14]. This is the rationale on the base of which cerebral monitoring is recommended [11] as a measure to reduce POD incidence. Temperature monitoring is also recommended, being hypothermia another risk factor for postoperative complications, POD included. In major surgery, non-invasive haemodynamic monitoring can be useful in guiding intraoperative fluids administration and preserving haemodynamic equilibrium (Goal-Directed-Therapy). Another important aspect related to anaesthesia is that, whatever technique is applied, optimal analgesia should be ensured, as pain is another causative factor for POD.

The presence of delirium should be investigated starting in the recovery room, using validated scores such as CAM or Nu-Desc. Residual effects of anaesthesia should be differentiated by POD using specific tests such as the Richmond Agitation Sedation Score (RASS), in order to allow prompt diagnosis of hypoactive delirium forms.

\section{Postoperative measures}

Postoperative management should focus on detecting POD every six hours, for a minimum of three-five days. Whenever possible, patients at risk of or under POD should be allocated in quite rooms where audio/visual stimuli are reduced. Right night/day rhythm should be ensured by general and/or pharmacological measures, avoiding the use of sedatives. Benzodiazepines are formally contraindicated in patients at risk for POD and their use should be only reserved to patients with extreme anxiety and/or alcohol withdrawal disorders.

Postoperative pain should be carefully investigated, mostly in cognitively impaired and demented patients. When traditional pain scales cannot be applied, dedicated scales such as PAINAD or NOPPAIN should be used.

Other postoperative measures include prosthetic aids availability, early mobilization and nutrition and measures aimed to preserve homeostasis.

\section{POD treatment}

As soon as signs of disorganized thinking appear, the patient should be checked to exclude any factor promoting POD (pain, bladder distension, intestinal occlusion, dehydration, glycemia imbalance or other 
disturbing circumstances). Adequate brain and other organs perfusion should be maintained, mostly in case of need for acute fluids replacement.

At the same time, non-pharmacological measures should be undertaken, such as re-orienteering strategies (availability of clock and calendar, frequent gentle recalling about surrounding circumstances) and normalization of sleep-awakening rhythm.

When these measures show to be ineffective and no elements disturbing homeostasis have been intercepted, pharmacologic measures should be applied. Low doses of intravenous or oral haloperidol $(0.25 \mathrm{mg}$ stepwise to a maximum of $3.5 \mathrm{mg}$ per attempt and to a maximum daily dosage of $6 \mathrm{mg}$ ) should be administered [11]. Given its cardiac side effects, caution should be applied in case of prolonged Q-T interval. Lowdose atypical neuroleptics showed to be also effective.

\section{Final considerations}

POD occurrence seems mostly influenced by the quality of care older patients receive. Dedicated clinical pathways such as Acute Care of the Elderly (ACE) Units or Proactive Care of Older People undergoing Surgery (POPS) are effective in reducing POD rate.

Adequate knowledge about both predisposing and precipitating risk factors, coordination among care team members and careful assistance are fundamental elements for its prevention, detection and management.

Given the scarcity of guidelines at European level focusing on perioperative care of older patients, and being shared culture and optimal organization indispensable ingredients for adequate geriatric care, there is a need for a strong cultural advancement in the field of geriatric surgery, anaesthesia and nursing.

Educational initiatives should be encouraged at University and non-University levels, and knowledge on POD incremented among professionals, in the interest of an increasing group of vulnerable and frail patients.

\section{References}

1. Bilotta F. Routine management of postoperative delirium outside the ICU: Results of an international survey among anaesthesiologists. EJA, in press 2016.
2. Publications Office of the European Union, European Commission: Demography Report. Short Analytical Web Note 2015.

3. Stagno D, Gibson C, Breitbart W. The delirium subtypes: a review of prevalence, phenomenology, pathophysiology and treatment response. Palliat Support Care 2004; 2:171-179.

4. Godoy Monzón D, Iserson KV, Jauregul J, et al. Total hip arthroplasty for hip fractures: 5-year follow-up of functional outcomes in the oldest independent old and very old patients. Geriatr Orthop Surg Rehabil 2014;5:3-8.

5. Crocker E, Beggs T, Hassan A, et al. Long-term effects of postoperative delirium in patients undergoing cardiac operation: a systematic review. Ann Thorac Surg 2016;102:1391-9.

6. Scholz AF, Oldroyd C, McCarthy K, et al. Systematic review and metaanalysis of risk factors for postoperative delirium among older patients undergoing gastrointestinal surgery. Br J Surg 2016;103:e21-8.

7. Inouye SK, Charpentier PA. Precipitating factors for delirium in hospitalized elderly persons. Predictive model and interrelationship with baseline vulnerability. JAMA 1996;20;275:852-7.

8. National Institute of Health and Care Excellence (NICE). Delirium: prevention, diagnosis and management. Clinical guideline [CG103], 2010. Available from: https:/www.nice.org.uk/guidance/cg103

9. American Geriatrics Society Expert Panel on Postoperative Delirium in Older Adults. American Geriatrics Society abstracted clinical practice guideline for postoperative delirium in older adults. J Am Geriatr Soc 2015;63:142-50.

10. Aldecoa C, Bettelli G, Bilotta F, et al. European Society of Anaesthesiology evidence- and consensus-based guidelines on postoperative delirium. EJA 2017 (in press).

11. Bettelli G, Ferrari A, Costantini M. Models of care and organizational solutions for geriatric care. In Perioperative care of the elderly: clinical and organizational aspects. G. Bettelli Ed. Cambridge University Press (in press).

12. Saczynski JS, Marcantonio ER, Quach L, et al. Cognitive trajectories after postoperative delirium. N Engl J Med 2012 5; 367:30-9.

13. Witlox J, Eurelings LSM, de Jonghe JFM, et al. Delirium in elderly patients and the risk of postdischarge mortality, institutionalization, and dementia: a meta-analysis. JAMA 2010;304:443-51.

14. Guenter U, Riedler R, Radtke FM. Patients prone for postoperative delirium: preoperative assessment, perioperative prophylaxis and postoperative treatment. Cuu Opin Anaesthesiol 2016;29:384-90. 